\title{
Le théâtre en Egypte : traces et trajectoires
}

\author{
Névine Sarwat \\ Département de Français \\ Faculté de Pédagogie \\ Université de Damanhour
}

مجلة الدراسات التربوية والاسانية ـ كلية التربية ـ جامعة دمنهور

المجلد الخامس العدد (4)- الجزء الثالث- لسنة 2013 


\section{Le théâtre en Egypte : traces et trajectoires}

\section{Le génie du poète et le talent des interprètes se}

Névine Sarwat reconnaissent justement à ce pouvoir d'imposer l'existence des personnages et l'univers moral dans lequel ceux-ci respirent et vivent leur drame ${ }^{1}$

L'art dramatique fascine tout le monde aujourd'hui par ses représentations, ses acteurs, ses thèmes, et nous pousse à nous demander où en est le théâtre égyptien ? Quelle place occupe- t- il ? Est-il le miroir d'une culture ? Comment fonctionne la grammaire de sa construction ? Comme tout, le théâtre, l'auteur, l'acteur et le spectateur doivent parler un langage, alors quel langage impose ce théâtre dans ce monde en pleine mutation?

Donnant une large place à l'étude de sa formation, de son épanouissement, de son développement, la présente étude essaye de disséquer l'ancrage de ce théâtre dans la vie sociale, pour créer son identité, sans bien sûr oublier sa naissance et ses origines françaises.

Entre le «dit» et le «non-dit», le théâtre égyptien entreprenait ses premiers pas. Un théâtre édifiant, et installant sur des places publiques ses premiers jalons, oblige le public à reconnaître sa valeur.

La révolution égyptienne du 25 janvier 2011 trouve ses origines dans ce théâtre. Plusieurs auteurs et acteurs se sont insurgés pour tracer une nouvelle voie pour l'Egypte. Cette entreprise prend en considération le rapport des pièces de thêâtre et du public pour forger une identité culturelle nouvelle.

${ }^{1}$ GOUHIER Henri, Le théâtre et l'existence, Librairie philosophique, J. Vrin, France, 1973, p.32, pp.224

653 
Dès l'aube des années 80 et l'apparition du théâtre dans le secteur privé, la révolution couve en Egypte, en donnant un sens à la création d'une volonté de dénonciation sociale.

Et Mohamed Safaa écrit,

«Les dernières 30 années ont permis à la révolution du 25 janvier de changer les conceptions et les modalités de la vie des égyptiens, aussi, fût- il nécessaire d'un changement radical dans la vie du citoyen égyptien afin qu'il puisse avoir sa liberté sans violer la liberté de l'autre. » ${ }^{2}$

La présente étude esquisse un itinéraire pour poursuivre le trajet du théâtre égyptien, afin de pouvoir analyser de près, comment le théâtre a joué un rôle important dans la vie des égyptiens, et comment a-t-il pu inciter le peuple à s'insurger pour aboutir à cette révolution.

Nous nous sommes interrogée sur la nature du théâtre égyptien, qui croise son public sur certains chemins privilégiés. Comment eut lieu sa naissance ? Pour y répondre, esquisser un profil historique est certainement nécessaire !

Nul ne peut nier les empreintes de l'occident sur la formation du théâtre égyptien. En effet, certains prétendent faussement que notre théâtre est issu de la période des pharaons. Or Dr. Abdel Moty Shaarawi avance qu'il n'y avait ni lieu ni démocratie permettant l'apparition d'un théâtre pharaonique, "Les pharaons ne possédaient pas de lieu pour les pièces de théâtre" 3

\footnotetext{
2 محمد صفاء، ثورة تعيد صياغة المسرح المصري أعمال جديدة تستلهم روح 25 يناير، جريدة المساء،

الجمعة 1 أبريل

2011

"ثورة 25 يناير استطاعت تغيير مفاهيم وأنماط حياة المصريين خلال "30 "عامأ مضت ومن ثن ثم لابد من تغيير

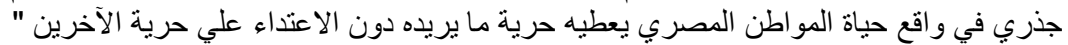

«La révolution du 25 janvier a pu changer les concepts et le mode de vie des égyptiens acquis durant les 30 dernières années, d'où la nécessité d'un changement radical dans la vie du citoyen égyptien lui permettant une certaine liberté sans pour autant attaquer la liberté des autres ».

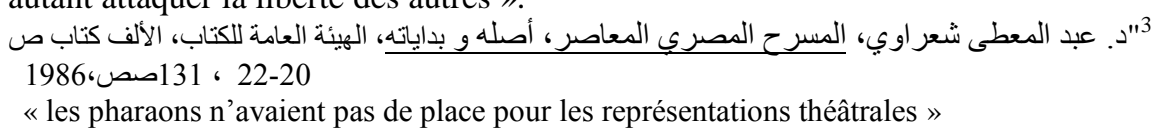

doi: $10.12816 / 0004236$ 
et plus loin, l'auteur assure que l'existence d'un théâtre ne peut naître que si un terrain où la démocratie existe,

"Si la démocratie disparaît, l'auteur dramatique ne peut pas s'exprimer et l'apparition des pièces de théâtre devient alors une impossibilité. C'est pour cette raison que l'écrivain pharaonique ne pouvait pas mettre en scène les différentes légendes comme Isis et Osiris";

En tant qu'art vivant sous le système du pouvoir pharaonique, le théâtre n'a pu ni naître ni voir le jour.

Les recherches antérieures nient le fait que le théâtre soit le facteur permettant le passage entre les différentes civilisations en Egypte. Néanmoins Jacques Berque écrit, dans Les arts du spectacle dans le monde arabe depuis cent ans,

«le théâtre d'ombres, les farces volontiers obscènes de Qaraqûz, la récitation de chansons de gestes, comme celles du cycle d'Abû Zayd al Hilâli, qu'on peut encore entendre dans les cafés du Vieux Caire, la veine mimique et parodique dans bien des manifestations populaires furent scrutés en tant que pouvant « conduire » à des formes modernes de l'art, analogues aux nôtres. Mais ces recherches ressortissaient généralement au folklore $\gg 5$.

D'après Berque, le théâtre se limitait aux scènes précédentes. Nous ne pouvons pas oublier que l'Islam a également eu son rôle délimitant les productions afin de respecter les principes

" لم يكن لدى الفراعنة أماكن للعروض الدسرحية "..

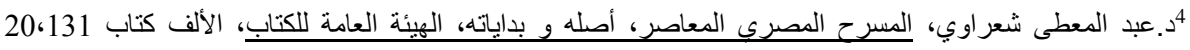

'Si la démocratie disparait, il sera difficile d'exprimer l'opinion du dramaturge. C'est pourquoi il sera difficile également qu'un art dramatique apparaisse. C'est pour cette raison que l'art dramatique pharaonique n'a pas pu voir le jour facilement. Le dramaturge pharaonique n'a pas pu traiter la légende d'Isis et d'Osiris ni toute autre légende sur le théâtre. »

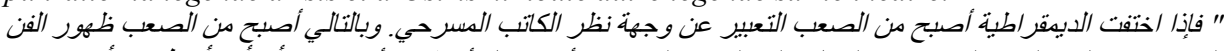

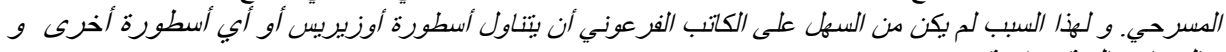

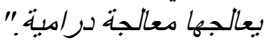

${ }^{5}$ BERQUE Jacques, Les arts du spectacle dans le monde arabe depuis cent ans in Le théâtre arabe, Unesco, Belgique, 1969, 229p., (pp.15-38), p.16

655 
de la foi islamique sans les transgresser. En dépit de l'existence d'une culture, qui se voulait une culture sans frontières Bencheneb Rachid souligne,

« la culture arabe moderne reflète une double préoccupation : l'attachement aux vraies valeurs arabo-islamiques et l'ouverture sur les littératures occidentales, principalement la littérature française $»^{6}$.

C'est dans ce contexte que la période révolutionnaire de Orabi et celle de l'occupation étaient à l'essor de la naissance de cet art et de ses représentations lors de l'arrivée de l'expédition française. Malgré le désarroi moral de ce peuple, il était assoiffé d'art.

De plus, l'expéditeur "La Jonquière"7 parlant du temps de l'expédition française, écrit que le Courrier d'Egypte a annoncé dans son numéro du 21 Octobre 1798, l'inauguration d'un club de lecture crée par Dargeval. Ce club n'était pas seulement un lieu de rencontre pour les écrivains et l'élite étrangère de la société, mais il se voulait un lieu de divertissement pour les soldats de Napoléon. Les acteurs des catégories de deuxième et troisième degrés venaient pour présenter sur scène cet art déjà connu en France depuis le XVème siècle.

Même après le départ de Napoléon, pendant la puissance de Kléber, les scènes ne manquaient pas de représentations. Afin de divertir ses soldats, Napoléon lui a envoyé une lettre pour assurer la présence du drame français sur les scènes égyptiennes afin de changer les coutumes des égyptiens.

Mais comment Napoléon voulait-il faire une modification dans la vie de ces derniers alors que sa présence était refusée?

\footnotetext{
${ }^{6}$ BENECHENEB, Rachid, Les sources françaises du théâtre égyptien, in Revue de l'Occident musulman et de la Méditerranée, N. 8, 1970, pp.9-23, p.10

${ }^{7}$ LA JONQUIÈRE, L'Expédition d'Egypte (1798-1801), Tome III, éditeur militaire, Paris, p.382
}

doi: $10.12816 / 0004236$ 
Minou, le successeur de Kléber, avait la même intention que son prédécesseur, il a construit à son tour le «Théâtre de Joumhoriah pour les arts $\gg 8$ pour la présentation des scènes théâtrales. L'ère de Mohamed Ali Pacha ne manque pas à la célébration des constructions théâtrales, les scènes, elles aussi, ont joui des pièces: L'avocat Patelin, Le Castronome sans Argent. La finalité de l'art du théâtre en ce temps devait viser le progrès.

Gérard de Nerval mettant l'accent sur l'évolution du théâtre, assure dans ses écrits, l'existence d'un théâtre intitulé « Teatro del Cairo » où il a passé une soirée agréable. Jusqu'à ce temps-là, la plupart des spectateurs, étaient soit des italiens soit des grecs. Les égyptiens étaient complètement absents à ces représentations, faute de langue et de culture.

L'avènement du théâtre en Egypte en langue arabe est sans doute une représentation d'une nouvelle ère d'épanouissement comme si les égyptiens faisaient appel à la scène, à un nouveau devenir scénique, à une nouvelle existence.

Dans sa trajectoire, le théâtre en Egypte a passé par trois phases :

La première période 1870-1904: les pionniers du théâtre égyptien

La deuxième période 1905-1939: la troupe de Salama Hegazi et de Georges Abiad

La troisième période :

« De Nasser vers la révolution du 25 janvier 2011 »

Dans un contexte où tout est incertain, ce n'est qu'à travers l'articulation politique du pays que le théâtre égyptien prendra forme.

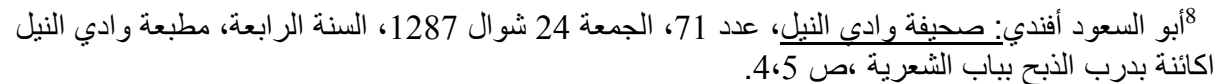


La première période 1870-1904: les pionniers du théâtre égyptien

L'ère de Saïd connut une renaissance théâtrale. A la fin de 1870 , les théâtres égyptiens connaissent pour la première fois des pièces jouées en arabe classique afin de pouvoir divertir les nobles égyptiens.

Les manifestations de cette évolution culturelle de 1769 à 1848 ouvrent le terrain, à la venue de James Sanua et de Salim neveu9 de Mârûn al -Naqqâsh », avec la « Nahda » que Tahtawi a fait découvrir à L'Egypte. Alain Mourgue confirmant cette renaissance qui imprégna la littérature égyptienne en ce temps, avance que

«La première délégation envoyée à Paris en 1826 est conduite par le savant français Jomard qui avait participé à l'expédition de Bonaparte. La délégation s'élève à 44 personnes et compte dans ses rangs quatre étudiants d'origine arménienne. Tous ces étudiants, musulmans et chrétiens, vont jouer un rôle déterminant dans le processus de la renaissance culturelle arabe : La Nahda (le réveil). L'émergence d'une littérature nouvelle - plus particulièrement en prose romanesque, historique et sociale illustre ce profond mouvement de renaissance de la culture arabe dans un monde en pleine évolution, dominé par la puissance des grandes nations occidentales qui se lancent dans l'aventure coloniale. $\gg 10$

Ainsi influencés par la culture française, ces deux grands écrivains, ont pu tracer un trajet scénique non négligeable aussi bien au Liban qu'en Egypte. A l'assouvissement de cet

9

${ }^{10}$ MOURGUE, Alain, L'émergence de la littérature arabe moderne en Egypte, la Nahda, in Revue trimestrielle Al Moukhtarat $n^{\circ} 45 / 46$ (année 2000) éditée par l'Institut du Monde Arabe, qui a consacré un article intéressant dédié aux poètes de la

doi: $10.12816 / 0004236$ Nahda, 2007, pp.1-11, p.3

658 
art, Naqqâsh, en 1847, donne une représentation en arabe de L'Avare, sa première pièce. Et, Bencheneb avance, « du Liban où une comédie de Mârûn an-Naqqâsh, inspirée de L'Avare de Molière marque en 1847 son entrée dans la littérature arabe, il émigre rapidement en Egypte, celle d'Ismâ'̂̂l (1837-1895), qui a choisi la France comme partenaire privilégié. Amateur de théâtre et de musique, le Khédive encourage les troupes françaises et italiennes qui acclimatent sur les bords du Nil le drame et l'Opéra $\gg 11$.

Etant l'expression dramatique, le théâtre de ce temps était régi par la loi de l'imitation, Ali El Ra'i.12

«Imiter pour reproduire » tel a été le slogan du théâtre en cette époque. Le théâtre de Qabbâni est remarquable grâce à sa conception originale : Bencheneb, décrit sa technique ainsi «des scènes violentes et pathétiques, des morceaux de bravoure, de belles tirades à portée édifiante, qui est suivie d'un récital de chant, de danse, de farce et de jeux de cirque $\gg 13$.

Connu par trois pièces empruntées à des sources françaises, Lucia ou Hîyal an-nisa'a (les ruses des femmes), Afîfa (La chaste), tirée de Geneviève de Barbarant et Mithridate de Racine, Qabbâni a toujours essayé d'avoir sa propre vision pour ses pièces. Il n'a gardé de la tragédie de Racine que le nom « Le Mithridate »14, Bencheneb révèle dans son étude que cette pièce « tient du mélodrame autant que de la parade et du cirque $\gg 15$. Influencé par des auteurs dramatiques français, il peint ses pièces par les pinceaux de Molière, de Racine et de Corneille. Il était fidèle en traduisant les

\footnotetext{
${ }^{11}$ BENECHENEB, Rachid, Les sources françaises du théâtre égyptien, in Revue de l'Occident musulman et de la Méditerranée, N. 8, 1970, pp.9-23, p.11

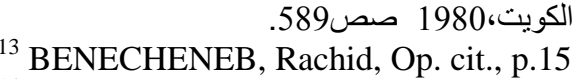

${ }^{14}$ Ibid., p. 16

${ }^{15}$ Ibid.
}

659 
différentes œuvres « il en a transposé les sujets dans la société égyptienne de son temps, avec ses croyances, ses coutumes et ses usages. »16. Il ne faisait que des adaptations en modifiant les noms, les costumes et le langage et gardant la structure originelle, pour que tout soit approprié à l'égyptien.

Avec l'avènement du Khédive Ismaïl l'Egypte sera témoin de la construction du « Théâtre de la comédie » en 1868. Les œuvres de Molière se voient jouer sur scène par les troupes de la Comédie Française. Un an plus tard lors de l'inauguration du Canal de Suez. En 1869, Ismail crée le théâtre de l'Opéra connu sous le nom de "Dar el Opéra" où une saison française et une saison italienne y sont périodiquement organisées.

« Yacoub Sanou », un second amoureux du théâtre français, dramaturge a étudié plusieurs auteurs comme Goldoni et Sheridan, Molière, qui ont influencé de près ses œuvres. Dr. Farouk Abdel Kader, dans son livre L'aube du théâtre égyptien17 souligne que lui-aussi comme Mârûn en-Naqqash est un fidèle adepte de Molière. Par les touches des italiens, des anglais et surtout des français, son théâtre a pu illuminer la société égyptienne.

Après les trois traductions des œuvres de Molière : Le malade Imaginaire, l'Avare et Tartuffe, Sanou a vécu des réussites successives qui lui ont valu le nom de « Molière d'Egypte ». Possédant les critères du théâtre occidental, il fascina le public de son temps, mais il faut mentionner que son goût et son penchant pour les chansons folkloriques, lui ont ouvert un vaste champ pour sa dramaturgie. La contiguïté de la Commedia dell'arte et le style folklorique ont donné naissance à un art qui touche le cœur du peuple.

Cet écrivain a pu présenter le caractère du "valet" en lui définissant une identité folklorique pour susciter l'amour et la

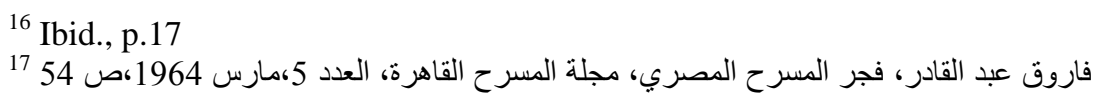

doi: 10.12816/0004236 
pitié du peuple citant à titre d'exemple "Abou Ridah el Barbary", le valet nubien typique, fidèle à ses maîtres. Maîtrisant son art, Sanou est allé plus loin en adoptant le dialecte arabe. "Le dialecte arabe » était son atout pour plaire au peuple égyptien. Ali El Ra'i souligne, «son mérite fut précisément de s'engager dans une toute autre voie. Il écrivit ce qu'il appelle lui-même une pièce lyrique en un acte" 18.

Étant de père chrétien, de mère juive qui l'avait confié à l'Islam pour lui éviter le sort de ses frères qui subissaient une mort subite, cette triade théologique le poussa vers un art dramatique propre au fonctionnement et à la puissance créatrice de son théâtre. Ces pièces ont pu être considérées comme les premières œuvres dramatiques du théâtre authentiquement égyptien. Il voulait voir une Egypte « Occidentalisée ». Avec une nouvelle conception de la vie et de l'art théâtral, il établit un contact étroit avec les gens du peuple, surtout en parlant leur idiome. Le Molière d'Egypte a imité l'idée de Molière «celle de former des acteurs types comme dans sa pièce Les tourments du Molière d'Egypte 19. Son hardiesse est allée jusqu'à s'en prendre au gouvernement et de dénoncer certains défauts de la société: critique du roi et de ses relations immorales et illicites, la polygamie, tels sont les thèmes qui touchent de près les égyptiens. Ceci lui a valu la fermeture de son théâtre et son expulsion de l'Egypte, tout en gardant le titre de «Molière d'Egypte» discerné par le Khédive qui lui dit ;

« Vous avez initié notre peuple à l'art du théâtre ; on vous appellera désormais le Molière d'Egypte, ».20

18 EL RAI, Ali, Le génie du théâtre arabe des origines à nos jours, in le théâtre arabe, Unesco, 230p. 1969,p.84

$$
\text { دار الثقافة بيروت نجم ـ مقدمة (في) يعقوب صنوع (أبو نضارة) ـ سلسلة المسرح العربي : دراسات ونصوص - } 1963 \text { ـ ص. }
$$

661 
A l'instar de son maître Molière, Sanou répond à ces critiques par son œuvre, Les tourments du Molière d'Egypte, comme Molière l'a fait avec "L'impromptu de Versailles, écrit en 1663 et Porseau où Molière critique Molière "l'auteur" et surtout sa pièce Les précieuses ridicules.

Une autre source française alimenta le théâtre égyptien. La troupe d'Abou Khalil Al Qabani, en 1885, présenta sa première saison théâtrale, en s'inspirant des pièces de Racine. La troupe d'Iskandar Farah qui avait pour héros Salama Hegazi présenta ses pièces les plus célèbres de 1891 à 1905.

Fasciné lui aussi par le théâtre Moliéresque et par ses procédés comiques, Mohamed Galal Uthman, traduit et adapte les pièces de Molière comme, L'Ecole des femmes, L'école des Maris, Les femmes savantes et enfin Tartuffe. Cette dernière pièce l'invita à égyptianiser son thème ainsi que les noms des personnages même si cela lui a coûté de s'éloigner complètement de la version initiale21."S'il lui arrive parfois de modifier une situation, écrit Becheneb, il le fait pour des raisons d'ordre religieux ou politique.

Durant cette période, dévoiler les vices de cette société occupait le premier rang des intérêts des dramaturges. La pièce"عصفور في القفص" un oiseau en cage: où Mohamed Taymour discute de la relation père - fils tout en mettant en évidence l'autorité du père sévère, la frustration de Hassan, le fils insurgé par cette domination, reflétant ainsi le conflit entre les générations, qui est au centre des préoccupations de Mohamed Taymour.

En effet, Taymour a pu présenter un théâtre simple en touchant par ses présentations les différents thèmes traitant des relations familiales. Cet auteur a pu jouer avec les différents registres de la langue pour pouvoir communiquer efficacement avec les différentes classes sociales. ${ }^{22}$

doi: $10.12816 / 0004236$ 
1.La deuxième période 1905- 1939: Salama Hegazi et de Georges Abiad

Dès 1905, la troupe de Salama Hégazi prend son élan sur les scènes égyptiennes. L'année 1914 vît un recul pour le théâtre dramatique alors que le théâtre comique s'épanouissait, réalisant de grands succès, surtout les troupes de Naguib El Rehani et de Ali El Kassar.

La première guerre mondiale jouait à son tour, un rôle très important pour la formation de l'identité du théâtre égyptien. Venant de la Syrie, le Cheikh Salama Hegazi avec Georges Abiyad, qui a eu sa formation théâtrale en France, formèrent « la troupe Abiyad et Hegazi ». Encouragé par le khédive Abbas, l'ère du théâtre de Hegazi, était prospère par le nombre des pièces présentées.

Le vaudeville en Egypte fît son apparition par la présence de Rehani, qui n'était en ce temps qu'un acteur descendant des Casinos de la rue Emad El Dine.

Réhani, de formation française, créa une troupe portant son nom. Ayant un goût pour la comédie, il présenta des personnages issus de la comédie française comme "Keshkesh bey le maire de Kafr el Balass". Doué pour le théâtre dès sa naissance, il joue comme Molière dans ses pièces, il a eu recours à Badii Khairi pour lui écrire le zajal, forme poétique en prose"الزجل"et c'est à travers ses pièces qu'il a essayé d'exposer les problèmes et les défauts de sa société en y insérant des chansons citant comme exemple sa pièce Eich , "اش l'hymne de la liberté dans sa pièce "Raene", "رن".Il fit une adaptation de la pièce de Topaze connue sous le titre de "La livre égyptienne".

Le second adversaire de Rehani ou plutôt son deuxième concurrent est Ali El Kassar. Directeur de troupe et acteur, ce dernier présenta "Uthman Abdel Basset" égaré dans la ville où il succombe au charme des belles danseuses étrangères comme le mentionne Becheneb. 
Tous deux, aimant le théâtre, entrent en compétition en créant des pièces qui par leur titre communiquent entre elles. Citons comme exemple, Réhani, écrit/ rahet aleik/ "راحت

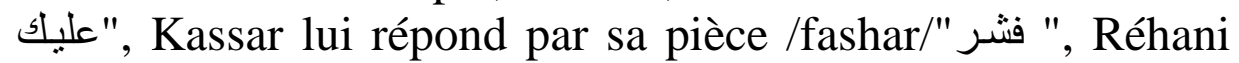
plus tard écrit / al seit wala el gheina/ "السيطو لا الغنى", Kasser lui répond par une autre pièce/walaou/ "و ول و qui fera un grand changement au théâtre égyptien.

A la troupe de Salama Hegazi, de Réhanni, de Kassar, vient s'accrocher la troupe de Fatma Rouchdi, de Georges Abyad et enfin celle de Youssef Wahbi incarnant le genre mélodramatique par excellence.

En 1911, le théâtre égyptien connaît avec Abiad une dimension plurielle dans la mesure qu'il y ajoute la dimension éducative à celle du divertissement, à la suite de la demande du Ministre de l'Education Saad Zaghloul qui voulait arabiser l'éducation. Abiad a répondu à Zaghloul que la meilleure voie était de commencer avec le théâtre.

L'indépendance de l'Egypte et la fin de l'occupation anglaise devinrent une nécessité alors une autre demande se révélait pour la création d'un théâtre national. Ce n'est qu'en 1921 que ce théâtre fut inauguré.

Attiré par le théâtre, le poète Khalil Moutrane, en 1935, déploya de grands efforts pour régler la crise du théâtre en payant des salaires aux acteurs sans revenus. «La troupe nationale égyptienne inaugura un institut pour les acteurs et envoya des missions à l'étranger. Mais en 1942, la troupe a été dissoute par décret. Quand la révolution éclata, il existait deux grandes troupes théâtrales: La troupe nationale égyptienne et la troupe égyptienne du théâtre moderne. »23

23

http://www.sis.gov.eg/Fr/Templates/Articles/tmpArticles.aspx?ArtID=819\#.UwIp4_m $\mathrm{SxHk}$

doi: $10.12816 / 0004236$ 
Citons à ce propos que Georges Feydeau était un des grands auteurs joués en Egypte par les troupes égyptiennes et était également source d'inspiration pour les pièces adaptées, comme la pièce de La dame de chez Maxim, adaptée sous le nom de "El pyjama el Hamara" et puis L'Hôtel du Libre échange adaptée sous le nom de "Lokandet El Fardouss".

La troisième période qui se divise en

«De Nasser vers la révolution du 25 janvier 2011 »

Le théâtre égyptien en général et le théâtre français en particulier, durant cette période, traitaient les problèmes humains et éthiques en amalgamant le patrimoine à la légende populaire. Encore une fois, l'importance de l'existence d'un théâtre qui représenterait l'esprit et la culture égyptienne s'imposa. Ainsi, il reflètera la vie réelle des égyptiens et en deviendra le miroir : la naissance du théâtre symbolique et réel égyptien entreprend son premier pas.

Dû à la vie politique et sociale dans les années 50, un nouveau théâtre émerge adoptant les problèmes politiques et sociaux comme thème majeur au lieu de les traiter comme un de ses composants thématiques. Nous assistons alors à une écriture qui trace son propre chemin.

L'Etat bien sûr n'a pas accepté ce genre de théâtre et les pièces s'orientaient plus vers le symbolisme, pour ne pas tomber dans le piège de la censure comme le fit notre grand auteur Tawfik El Hakim. Le goût comique a teint ses pièces pour satisfaire le goût d'un grand public. Ce grand écrivain a eu recours à des pièces, qui avaient pour origine le théâtre européen. Dans presque toutes ses pièces, il donnait une grande importance à la relation qui liait le gouverneur et le gouverné comme /Al hamir/ الحمير / et / Bank al kalak/ بنك القلق. Le dialecte égyptien, s'est imposé dans ce genre de pièces comme moyen pour dissoudre la différence entre les classes sociales, qui hissent le drapeau «socialiste». Le théâtre occidental comme ceux de Brecht, de Ionesco et de Beckett, marquent par leurs touches la peinture de ces pièces.

665 
Presque toutes ces pièces en traitant les problèmes politiques et nationaux optent pour un théâtre engagé, influencé par Jean Paul Sartre. Il faut souligner que la plupart des pièces de Hakim reflètent souvent ce goût de la dualité entre le rêve et la réalité. En vérité, son théâtre présente "le conflit entre les idées et stimule la réflexion"24 .

Noaman Achour, présente encore une fois le conflit existentiel dans la société égyptienne à travers la rivalité naissant entre le père et le fils et menant au conflit entre deux aspects sociaux: le traditionnel conservateur et le modernisme libéral. Ce conflit se présente sous une autre forme chez Saad El Dine Wahba qui s'appuie sur l'opposition entre les différentes classes sociales, comme dans sa pièce /Al sebensa/(السبنسة 1966) où il hiérarchise les classes sociales en 1ère , 2ème et 3ème classe. Et voilà le proviseur de la gare "Nazer el mahattah" qui crie aux passagers:

"Mettez-vous de côté.... la première classe de ce côté désignant le gouverneur et les soldats.

Et la deuxième classe par là- désignant Darwish et Fatthi Et la troisième classe ici - désignant les accusés et Saber » Plus loin, Saber répond:

« la bombe s'explosera, ...., la terre est pleine de bombes, notre pays a été planté de bombes et s'explosera et ceux de la première classe seront en troisième et ceux de la troisième en première $» 25$

Les péripéties de cette pièce ne sont qu'un miroir qui reflète la réalité amère de cette société où le despotisme et le tragique sont les premiers acteurs du village «Kafr El Akhdar » et «La bombe». Il en résulte la révolution qui se déclenchera un jour.

${ }^{24}$ BÉNCHENEB, Op. cit., p.23

${ }^{25}$ WAHBA, Saad el Dine, el Sébénssah السبنسسة - 1966.

doi: $10.12816 / 0004236$ 
S'interrogeant dans sa pièce sur l'intérêt des nouvelles lois socialistes, si elles ont vraiment pu sauver la société de l'injustice et de même ont-elles pu éliminer tous les vices ? Il aboutit au fait que le « socialisme » n'était qu'un régime pour que la nouvelle classe profite de la nouvelle situation politique du pays.

Noman Ashour, par ces deux pièces a pu répondre à une question qui se pose à la première pièce (Wabour el tahin) 1965, en soulevant le problème des opportunistes tout en utilisant le symbolisme réel الأسلوب الرمزي الواقعي alors que pour sa seconde pièce/belad bara/بـالداب بركي en 1967, il a pu résoudre la problématique en ayant recours au réalisme direct. Saad El Dine Wahba a traité le même problème dans sa pièce 1965, en exposant une nouvelle classe qui naît de l'héritage de /Al mahroussa/ l'ancien régime.

Le maire, parlant de la mère de Farid, au veilleur26, «Je vois qu'elle a des pigeons... et si elle proteste amène Farid avec toi....il a été engagé au service militaire». 27

De nouveaux principes émanent de cette classe comme l'égoïsme, l'hypocrisie et l'exploitation comme l'a bien décrit Youssef Idris dans sa pièce /al farafir/ «Al Farafir». Alfred Farag avance dans une étude sur « Al Farafir »,

Telle n'était peut-être pas son intention ; il a cependant voulu provoquer un «choc» dans les esprits, les tirer de leur stupeur, de leur somnolence ou de leur quiétude. On se souvient que l'objet tant de la littérature politique de l'époque que de la propagande intensive sur le socialisme et ses réalisations, portait principalement sur les détails et esquivait pratiquement le débat de fond sur l'essence de l'appel au

\footnotetext{
"العمدة للغفير" 26

${ }^{27}$ WAHBA, Saad el Dine, المحروسة, 1965

667
} 
progrès social visant à transformer radicalement le rapport entre puissants et humbles.28

Alfred Farag ajoutant dans son article « Youssef Idris : AlFarâfî̀ »,

«Vous ne serez pas étonnés de trouver dans cette préface (entendez aussi : «dans cette pièce »), des opinions qu'il vous semblera avoir déjà lues ou entendues : tel n'était pas le cas en 1964. La pièce représentait une innovation pour l'ensemble du théâtre arabe. Le seul fait de la défendre était alors considéré comme un acte de courage qui pouvait mériter à ses partisans de se faire trancher le cou; tout ceci aujourd'hui est banal. Et on ne s'étonne plus que cette opinion, impensable en 1964, se soit répandue et ai(sic) rallié les écrivains du Maroc, de Syrie, du Koweït ou de Tunisie et jusqu'aux tenants du théâtre commercial tel celui de Tahiyya Karioka... »29

La relation entre le gouvernant et le gouverné الحاكم و المحكومa a été bien tracée par Idris dans sa pièce el Farafir30, Noman dans/belad bara/ بلاد برة et Tawfik el Hakim, dans /ya talee al shagara/ ياطالع الشجرة

Le «gouverneur » dans le théâtre de Tawik el Hakim apparait avec des idées et des slogans qui soulignent son despotisme en l'absence de lois qui permettent de contrôler l'anarchie de la société. Le chauffeur de 《سكة السلامة /al mokhatetin/ 《 المخططين 1969 et Bahar dans /ya talee al

${ }^{28}$ Référence électronique

Alfred Farag, "Youssef Idris : Al-Farâfîr ", Égypte/Monde arabe, Première série, Perceptions de la centralité de l'Égypte 1, [En ligne], mis en ligne le 08 juillet 2008. URL : http://ema.revues.org/index1191.html. Consulté le 24 mai 2012.

${ }^{29}$ Ibid., Consulté le 24 mai 2012.

${ }^{30}$ Al-farâfîr, avait doté l'art dramatique d'une dimension nouvelle, non moins importante que les perspectives révélées par un petit nombre de pièces d'avant-garde.

doi: $10.12816 / 0004236$

668 
shagara/ ياطالع الثجرة « personnifient les mêmes traits de caractères du despote, le « gouverneur » de El Hakim.

Nous déduisons que «le théâtre réaliste socialiste » a son rôle important pour l'évolution du théâtre de point de vue de l'idée et du contenu de la pièce.

Les principales caractéristiques de ce théâtre :

Le héros frustré comme chez Gorki

Le discours chanté 31comme chez Abdel El Rahman el Charkawi, Salah Abdel Sabour, Noman Ashour, Youssef Idris, Lotfi El khouli et Alfred Farag.

L'introduction dans le théâtre de la tragi-comédie (la comédie noire) comme chez Saad el Dine Wahba et Noman Ashour.

Le mariage entre l'arabe classique et le dialectal comme exemple de la dissolution des classes sociales.

En effet le courant réaliste, est un des résultats de la révolution, qui a participé à la modification de l'esprit et de l'âme de toute une nation. Nous pouvons donc déduire que l'art théâtral a un but plus noble que celui de refléter les problèmes de la société.

C'est au début des années 60, qu'une nouvelle identité théâtrale se dessina, voulant à tout prix s'éloigner de tout ce qui est occidental, Dr. Tharwat Okacha a créé «l'Institut général de l'art théâtral et de la musique » afin d'affirmer l'identité égyptienne.

Tawfik Al Hakim par sa pièce /al aydi al naema / الأيدي الناعمة aux professeurs des universités comme Samir Sarhan, Mohamed Anani et enfin Fawzi Fahmi.

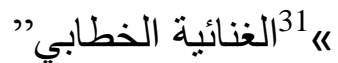


En 1972, en tant qu'auteur Linin el Ramli et Mohamed Sobhi en tant qu'acteur et metteur en scène, tous deux ouvrent la voie au théâtre privé par leur troupe « Studio $80 » 32$.

Par des séries de pièces s'enchaînant pour se compléter, Sobhi parvient à entamer ce type de pièces pour être parmi les pionniers de la révolution du 25 janvier. Tout un parcours, rien que pour pousser le peuple à dire "Non" face à la dictature. Cet acteur et metteur en scène, déploie des efforts pour ouvrir son éventail théâtral, qui sera témoin de son patriotisme.

Voulant défendre le pays contre les despotes, Sobhi adoptant un goût ironique présenta Carmen pour critiquer le dictateur et prévoir la révolution. Avec Mama Amrika, il imite l'exprésident, bien sûr en se moquant de lui pour le ridiculiser afin de le faire découvrir au peuple. Et ainsi dégarnir sa politique, prévoir et annoncer le futur en disant "nous nous attendons à des jours noirs!". Bel Arabi al Fasih en est un second exemple.

El Hammagy, Takharif et Weghat Nazar, permettent à Sobhi de dévoiler les vices de la société pour en faire une matière comique afin de pousser le peuple à s'en débarrasser.

Avec la volonté de défendre le pays contre le despotisme plusieurs auteurs et dramaturges ont hissé le drapeau de la révolution sur leur théâtre.

Nous allons essayer de présenter la pièce de Takharif de Sobhi et Elzaim, de Adel Imam avec plus de détails.

Sobhi avec un goût ironique critique le dictateur tout en préparant une révolution. Takharif est une pièce qui dévoile tous les vices de la société aussi bien économiques que politiques. Sobhi dans cette pièce joue le rôle du dictateur de la république Antika. Il contrôle tout. Ce dernier ne fait que

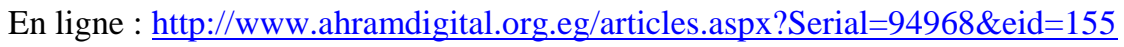

80 132

doi: $10.12816 / 0004236$

670 
promulguer des lois pour que son peuple se noie dans les dettes, les impôts, l'ignorance et la pauvreté. L'humiliation et la famine sont devenues la devise de cette république. Ne possédant pas le droit de se révolter, le peuple est soumis pour toujours et sera à jamais la victime de ce système dictatorial. Dans ce régime, le président nomme lui-même ses ministres qui servent ses intérêts, même si ces derniers sont au détriment de ceux du peuple. Les ministres seront choisis d'une manière égoïste pour se soumettre aux désirs du dictateur. Après un certain temps, le peuple s'insurge contre la situation pour ré-stabiliser la démocratie tant rêvée.

Ce message rêvé petit et court comme il est, était envoyé pour secouer le peuple égyptien.

La construction de cette pièce souligne la valeur de l'oppression d'un peuple ignorant et humilié. Sobhi personnifiant le président avec ses différents procédés comiques révèle la corruption de cette république.

En plein pessimisme et désespoir, nous pouvons voir une seconde pièce de Adel Imam, El Zaïm, qui à son tour dévoile l'injustice et ridiculise la société. Cet acteur incarne les leaders des pays arabes et les défauts de leurs systèmes politiques qui amènent le pays à une situation misérable. Il présente un second modèle du dictateur injuste, hypocrite, orgueilleux et vaniteux.

Ce tyran gaspille son argent pour satisfaire ses désirs. Le problème des élections est un des sujets discutés dans la pièce. L'injustice touche le système électoral, le droit de gagner les élections ne nécessite pas la voie parlementaire.

La pièce commence par la mort du leader d'un pays arabe. Présentant le rôle de Zinhom, le personnage pauvre, qui ressemble énormément au leader de ce pays, Imam (l'acteur) tombe dans le piège. Les proches de ce dernier lui tendent un stratagème pour qu'il puisse avoir toute la fortune. Ils lui ont demandé de jouer le rôle du leader défunt. Ayant accepté de faire partie de ce stratagème, il dévoilera les vices du système 
de Mobarak33. Dans une de ces scènes Adel Imam «Zinhom » utilise tous ses procédés pour amplifier les vices de l'ex-président et pousser le peuple à s'insurger. L'intonation et le jeu des mots étaient au service de Imam pour faciliter sa mission, pour accentuer son ironie noire et son refus total de ce régime.

«Zinhom » est allé jusqu'à prier pour l'ex-président en faisant des transgressions des normes langagières, mais en effet, les paroles n'engendrer qu'une signification, c'est de pousser le peuple à voir les défauts et les vices qui rongent l'infrastructure de cette société. Il faut que ce peuple se révolte.

Aujourd'hui comme jadis, la séparation entre politique et acteur est toute relative, ainsi nous prouve Farouk Goweida, poète éminent et écrivain de la pièce « Le khédive »34 . Définir l'injustice, comme on le fait souvent, par une prise de distance au théâtre est facile mais peut conduire à une ambigüité. Or Goweida a présenté sur scène ses idées dramatiques sans équivoque, une attaque du système politique du président Moubarak dix-huit ans avant que la révolution du 25 janvier ne se déclenche. Grâce à la plume de l'auteur, l'originalité et la force du thème, cette œuvre s'impose. Toutefois pour voir le jour, il a fallu attendre dixhuit ans pour échapper à la censure du temps de l'ex-président Moubarak.

Le discours se construit savamment à mesure que l'intrigue avance, la dynamique de l'action tient tout au long de la pièce à un face à face : oppresseur et opprimé, le public applaudit les héros en réalisant que rien ne sert de se taire, il faut briser

\footnotetext{
${ }^{33}$ Bien que Adel Imam (en tant que personne), était pour le système de Moubarak mais dans cette pièce, il est contre le système.

${ }^{34}$ GOWEIDA, Farouk, Le khédive, 1993 en ligne :

http://www.elcinema.com/work/wk1976985/
}

doi: $10.12816 / 0004236$ 
les barreaux de la prison, en se révoltant contre tout le système de Moubarak, les paroles deviendront indissociables de la notion de liberté. Vu les changements opérés récemment au niveau de la société égyptienne, la pièce devient une expression plurielle, ce qui lui donne une valeur importante après la révolution. 
«Si le symbole est connu et compris, c'est déjà de l'écriture. $» 35$

Un théâtre qui renvoie à la sensibilité d'une société et à son évolution, qui s'exprime aujourd'hui avec les mots d'aujourd'hui. Sa mission n'est plus de montrer mais d'ébranler, n'est plus de dévoiler mais de déstabiliser.

De ce lien, du mariage entre la liberté et l'écriture, nous pouvons déduire que la révolution a fait couler beaucoup d'encre, ainsi, la pièce «Lessons in Revolting»36, pièce jouée par les jeunes pour raconter le défi et le pouvoir d'un peuple. Vivre la réalité politique sur scène ou en scène, est devenu une passion pour tout auteur,

«A la fin de la pièce, public et acteurs se retrouvent aux tables du café qui jouxte le théâtre, autour d'un thé et d'une chicha, entre les voitures, capot ouvert, sur lesquelles travaillent jusqu'à tard dans la nuit les mécanos du garage d'en face. »37

Pour conclure, disons comme Jamila Mascat «faire la révolution, ça s'apprend sur le tas, et on a toujours beaucoup à apprendre ». Ainsi le théâtre égyptien à travers plus de cent ans a prouvé sa capacité d'assimiler les expériences du passé

${ }^{35}$ MARCEL, Mauss, Manuel d'ethnographie, Payot, 1967, p. 144.

${ }^{36}$ SOLIMAN, Laila est une metteur en scène et écrivaine égyptienne de trente ans. Ruud Gielens, trente-quatre ans, est belge, Cairote d'adoption, metteur en scène également et producteur. Tous deux sont à l'origine et codirigent Lessons in revolting/Dourous fil thaoura, une performance théâtrale d'une heure et demie qui a fait salle comble entre le 19 et le 23 août 2011 au théâtre Rawabet, situé dans une ruelle étroite et animée de Wast el-Balad, le centre-ville du Caire

37 MASCAT, Jamila, Révolution égyptienne, premier acte, http://www.slateafrique.com/32947/theatre-caire-tahrir-revolution-egypte-armee, consulté 01/09/2011 
et l'influence des différentes littératures, surtout la littérature française, et de se refondre selon une nouvelle méthode conforme aux évolutions survenues. Le Théâtre qui fut lancé en Egypte il y a cent ans, ne peut être considéré comme le miroir d'une société seulement mais bien plus il est devenu un outil pour lui forger son identité. Dans les pays arabes en pleine révolution, où la justice se fraye un chemin, le théâtre représente le porte-parole du peuple.

Al Ahram Hébdo, deux mois après la révolution, nous présente son article " La révolution sur les planches" écrit par May Sélim, pour confirmer que la révolution donnera naissance à des talents et à des artistes qui forgeront la nouvelle identité des planches égyptiennes. Citons à titre d'exemples les pièces suivantes :

El Balad baladna, (le pays est le nôtre) Charqi Charqi,(oriental, oriental) Eatessam Fanane (la grève d'un artiste) Soue? Tafahom pour critiquer les médias.

Enfin, un siècle de dramaturgie, nous ne pouvons pas nier que, le théâtre égyptien avait besoin de cette âme innovatrice, pour une nouvelle renaissance. En dernier lieu l'énergie de l'écriture scénique, l'enthousiasme, la fantaisie et l'humour des acteurs finiront par redonner espoir à un pays en mutation traqué par des conflits intérieurs et extérieurs. 


\section{Livres}

\section{Références :}

1.EL RAI Ali, Le génie du théâtre arabe des origines à nos jours, in le théâtre arabe, Unesco, 230p. 1969,p.84

2.GOUHIER Henri, Le théâtre et l'existence, Librairie philosophique, J.Vrin, France, 1973, p.32, pp.224

3.MARCEL Mauss, Manuel d'ethnographie, Payot, 1967, p. 144.

4.MIQUEL André, La littérature arabe, PUF, collection Que sais-je ? 1993.

5. عبد المعطى شعر اوي، المسرح المصري المعاصر ، أصله و بداياته، الهيئة

العامة للكتاب، الألف كتاب 22-131،20 ص، ص، ص،1986

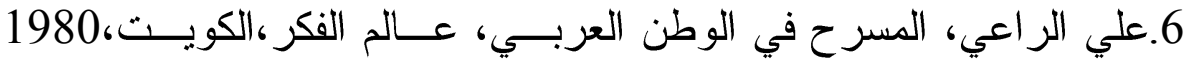

صص.589 - n

7.فاروق عبد القادر،فجر المسرح المصري، مجلة المسرح القاهرة، العـدد

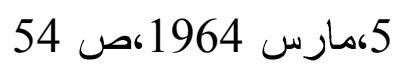

- Articles

1.أبو السعود أفندي: صحيفة و ادي النيل، عدد 71، الجمعة 24 شو ال 1287،

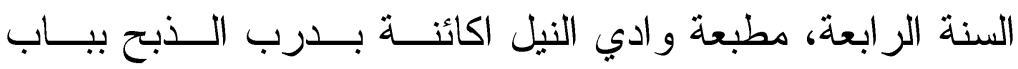

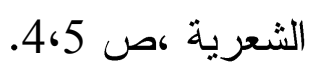

2.سامي منير حسين عامر (دكتور)، المسرح المصرى بعد الحــرب العالميــة

الثانية بين الفن النقا السياسـي والاجتمــــي (1945-1970)،

الجزآن، الهيئة المصرية للكتاب، الاسكندرية،1978

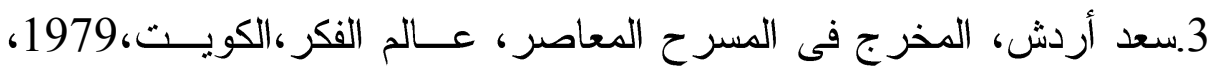

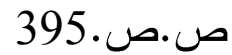


4.صبري عبدالعزيز، الرؤي التشكيلية فـي العــروض المســرح المصـرى

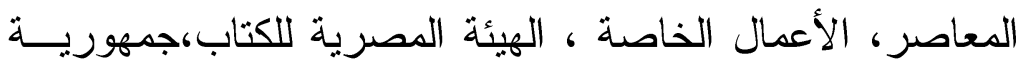

$$
\text { مصر العربية، 2002، ص.صناع.265 }
$$

5.فتحي العشرى، نبضات المسرح، المكتبة الأكاديمية،القاهرة ،جمهورية مصر الدر

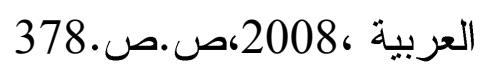

6.محمد صفاء، ثورة تعيد صياغة المسرح المصري أعمال جديدة تستلهم روح

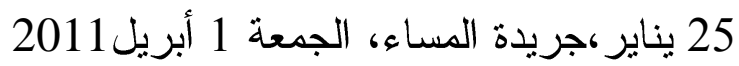

7.محد يوسف نجم - مقدمة (في) يعقوب صنوع (أبو نضــارة) - سلســلة

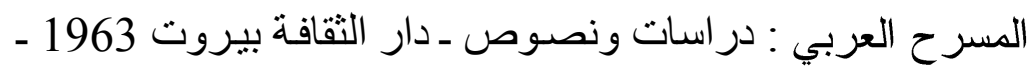<smiles>[Li][Ca]</smiles>

8.FARAG Alfred, «Youssef Idris : Al-Farâfîr », Égypte/Monde arabe, Première série, Perceptions de la centralité de l'Égypte 1, [En ligne], mis en ligne le 08 juillet 2008. URL: http://ema.revues.org/index1191.html. Consulté le 24 mai 2012.

9.Dans sa préface à la dernière édition (1988, éd. Maktabat Misr)

10.BERQUE Jacques, Les arts du spectacle dans le monde arabe depuis cent ans in Le théâtre arabe, Unesco, Belgique, 1969, 229p., (pp.15-38), p.16

11.BENECHENEB Rachid, Les sources françaises du théâtre égyptien, in Revue de l'Occident musulman et de la Méditerranée, N. 8, 1970, pp.9-23, p.10

12.LA JONQUIÈRE, L'Expédition d'Egypte (1798-1801), Tome III, éditeur militaire, Paris, p.382

13.MOURGUE Alain, L'émergence de la littérature arabe moderne en Egypte, la Nahda, in Revue trimestrielle Al Moukhtarat $n^{\circ}$ 45/46 (année 2000) éditée par l'Institut du Monde Arabe, qui a consacré un article intéressant dédié aux poètes de la Nahda. 2007, pp.1-11, p.3

\section{- Arabe}

doi: $10.12816 / 0004236$ 
1.بو السعود أفندي: صحيفةو ادي النيل، عدد 71، الجمعة 24 شوال 1287 128، السنة

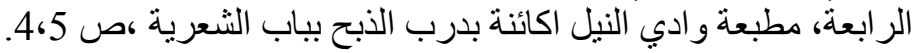

\section{webographie}

2.http://www.sis.gov.eg/Fr/Templates/Articles/tmpArticles.aspx?ArtID= 819\#.UwIp4_mSxHk

3.http://www.ahramdigital.org.eg/articles.aspx?Serial=94968\&eid=155

4.http://www.universalis.fr/encyclopedie/theatres-du-monde-le-theatreturc/5-le-theatre-d-ombres-du-karagoz/

\section{Pièces en ligne}

1.GOWEIDA Farouk, Le khédive, 1993 en ligne : http://www.elcinema.com/work/wk1976985/

2.MASCAT Jamila, Révolution égyptienne, premier acte, http://www.slateafrique.com/32947/theatre-caire-tahrir-revolutionegypte-armee consulté 01/09/2011. 
